\title{
ATLAS LUCID detector upgrade for LHC Run 2
}

\author{
Oleksandr Viazlo* on behalf of the ATLAS Collaboration \\ Lund University (SE) \\ E-mail: oleksandr.viazlo@cern.ch
}

\begin{abstract}
During the 2009-2013 data taking period (Run I) LUCID was successfully providing information about the luminosity delivered to ATLAS by the LHC. Starting from 2015 (Run II) the LHC machine is expected to provide about twice larger peak instantaneous luminosity and the bunch spacing in the machine is decreased by factor of two (from $50 \mathrm{~ns}$ to $25 \mathrm{~ns}$ ). The original LUCID design could not cope with the new running conditions, which would lead to saturation of photomultipliers and the luminosity algorithms, as well as problems with the lifetime of the photomultipliers. To address these problems a new LUCID detector was built and the readout electronic was redesigned. This article describes the design, the performance, new calibration system and the first results of $13 \mathrm{TeV}$ proton-proton collisions recorded by the new LUCID detector.
\end{abstract}

The European Physical Society Conference on High Energy Physics

22-29 July 2015

Vienna, Austria

${ }^{*}$ Speaker. 


\section{LUCID}

LUCID is a luminosity monitor with two detectors placed around the beam-pipe on both forward ends of the ATLAS detector. Each detector consists of 16 photomultipliers and 4 quartz fiber bundles. The photomultipliers (PMTs) detect charged particles that traverse their quartz windows, where Cherenkov light is produced. Cherenkov light is produced in the fiber bundles as well and carried to PMTs that are protected by shielding some 2 meters away (see Fig. 1). To increase the detector lifetime, only a subset of the PMTs is used at a given time, the others being available as spares. In addition, 4 PMTs have a reduced window opening to decrease their acceptance and thus avoid saturation of some luminosity algorithms.

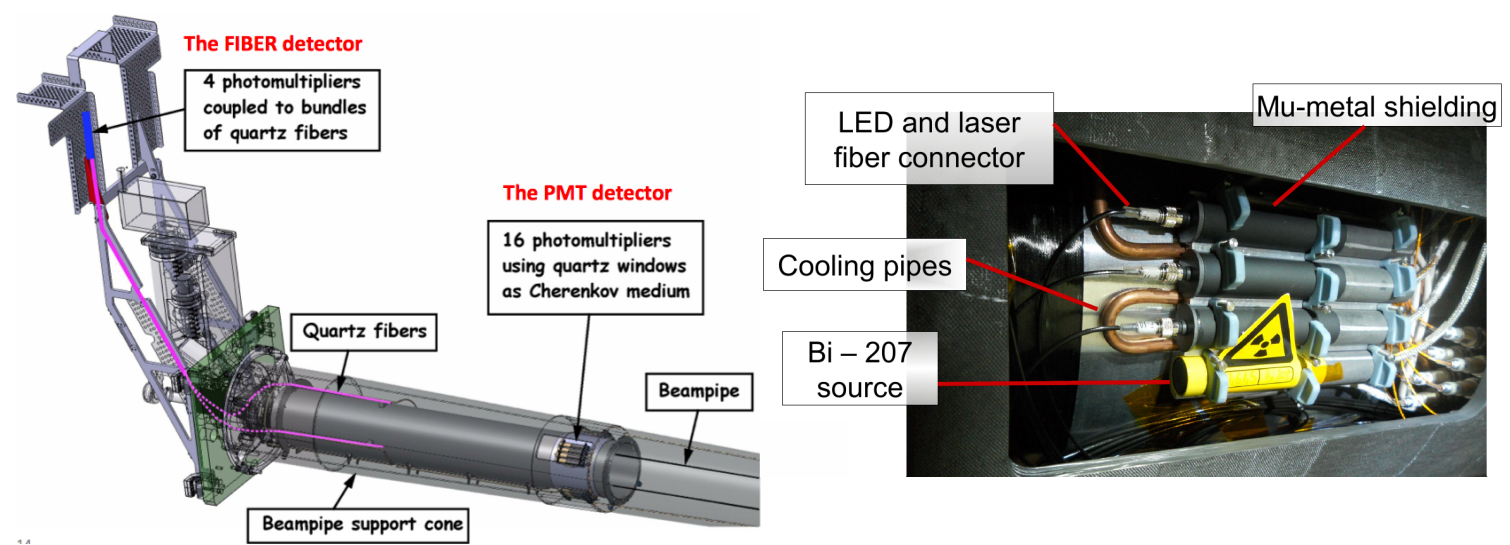

Figure 1: (left) Schematic drawing of one of the two detectors, showing the position of the photomultipliers and quartz fibers with respect to the LHC beampipe; (right) A quarter of one of the detectors. All tubes are placed inside mu-metal shielding to protect the PMTs from a stray field. Cooling pipes were installed in order to protect the PMTs from overheating during the beampipe bake-out procedure. Three of four tubes have fiber connectors, which tranfser LED and laser pulses for calibration. The fourth tube is equipped with a Bi-207 source and is completely sealed.

New readout electronics have been built that consist of VME boards that digitize the PMT signals with FADCs. The electronics record hits if the pulseheight is above a threshold and integrate the pulses in each 25 ns interval that correspond to a LHC bunch crossing. Figure 2 shows a typical PMT signal shape in a physics run. The duration of the pulses is less than $25 \mathrm{~ns}$. With respect to the detector used in Run I [1], the new LUCID has a reduced material budget, an increased dynamic range and will measure luminosity with additional algorithms based on PMT charge integration. It also has a completely new calibration system.

\section{PMTs and calibration system}

The new LUCID uses R760 Hamamatsu PMTs, a smaller version of the previously used R762 model. These PMTs have a $10 \mathrm{~mm}$ quartz window diameter, while the old ones had a $14 \mathrm{~mm}$ diameter. A smaller PMT model has been chosen to reduce acceptance which will help to cope with the increased occupancy and to avoid saturation of the luminosity algorithms. In addition, 4 PMTs per side have a specially reduced sensitive window with a $7 \mathrm{~mm}$ diameter which roughly 


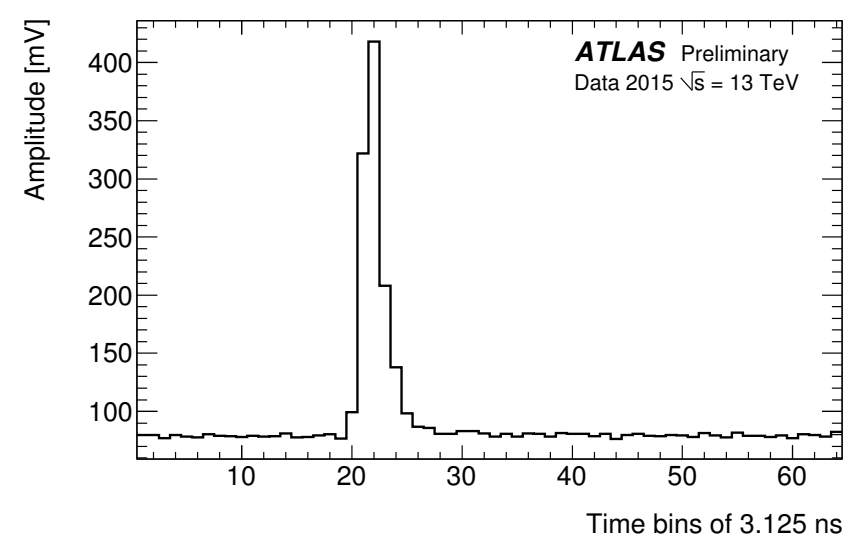

Figure 2: Digitized pulse shape of a signal from one of the PMTs of the LUCID detector during a run recorded on the 10th of June 2015 at $\sqrt{s}=13 \mathrm{TeV}$. The polarity of the pulse is inverted. The FADCs measure the pulse amplitude in time bins that are $3.125 \mathrm{~ns}$ long. Taken from Ref. [3].

corresponds to a factor 2 decrease in rate. They provide luminosity algorithms that will not saturate at increased luminosity.

The PMT gain calibration is monitored in 3 independent ways (see Figure 3):

- by LED signals carried by optical fibers;

- by laser signals transferred from the calibration system of ATLAS Tile Calorimeter;

- by radioactive sources (Bi-207).

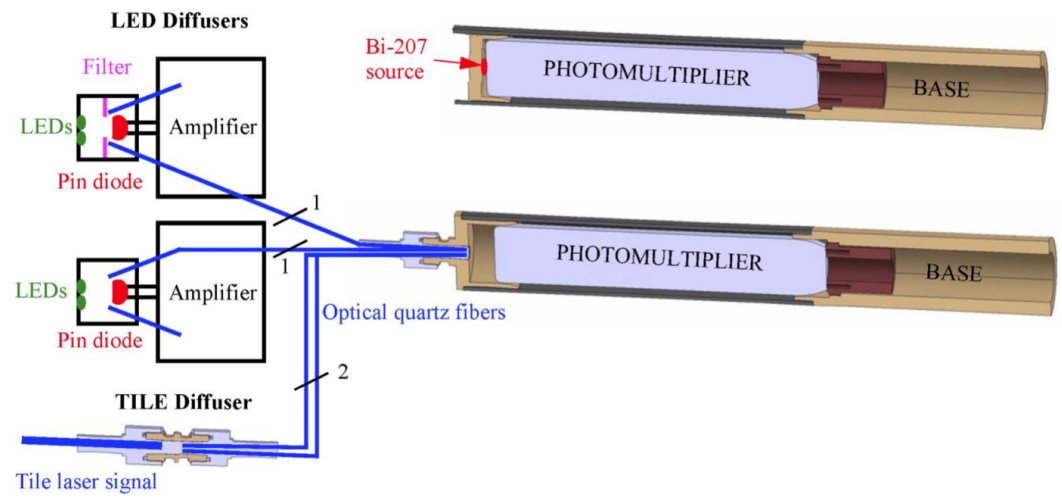

Figure 3: The LUCID calibration system. 16 PMTs per side receives light from LEDs and the Tile laser calibration system. For redundancy, two fibers come from two different LED diffusers (with three LEDs each, monitored by PIN-diodes), and two fibers come from one laser diffuser. The four remaining PMTs in each detector are calibrated with Bi-207 sources.

LED signals provide sharp peaks in the amplitude and charge distributions that are recorded by LUCID. The stability of the PMT gain is controlled by measuring the mean value of these distributions. The stability of the LEDs themselves is controlled by PIN-diodes. An alternative 
way of calibration is to use the ratio of the mean charge measured by PMTs with that of the PINdiode. This charge is proportional to the LED intensity and by controlling charge ratio allows to rule out any dependence of the calibration results on LED intensity fluctuations.

The Tile calorimeter laser system provides an alternative source of stable light and is treated in the same way as the LED signals in the calibration procedure. The stability of the laser signals is monitored by the Tile calibration system [2].

$\mathrm{Bi}-207$ radioactive sources provide monoenergetic electrons from an internal conversion process with energies above the Cherenkov threshold in quartz. These electrons have enough kinetic energy to penetrate the quartz window of PMT and produce signals similar to the signals from high energetic particles from physics runs (see Section 4). The mean of the charge and amplitude distributions from the Bi-207 sources are used in the same way as for the two methods described above. This method doesn't suffer from any instability issues.

The availability of three independent calibration methods increase the robustness of the calibration system and provide a possibility to cross-check calibration results between the methods.

Figure 4 shows a trending plot of Bi-207 calibrations during a 1.5 month period. There is no significant deviation from the nominal value. A $10 \%$ change of mean charge correspond to about a $1 \%$ change in luminosity. In case of a decreasing gain due to PMTs aging the high voltage is increased by an automatic procedure to keep the gain constant with time.

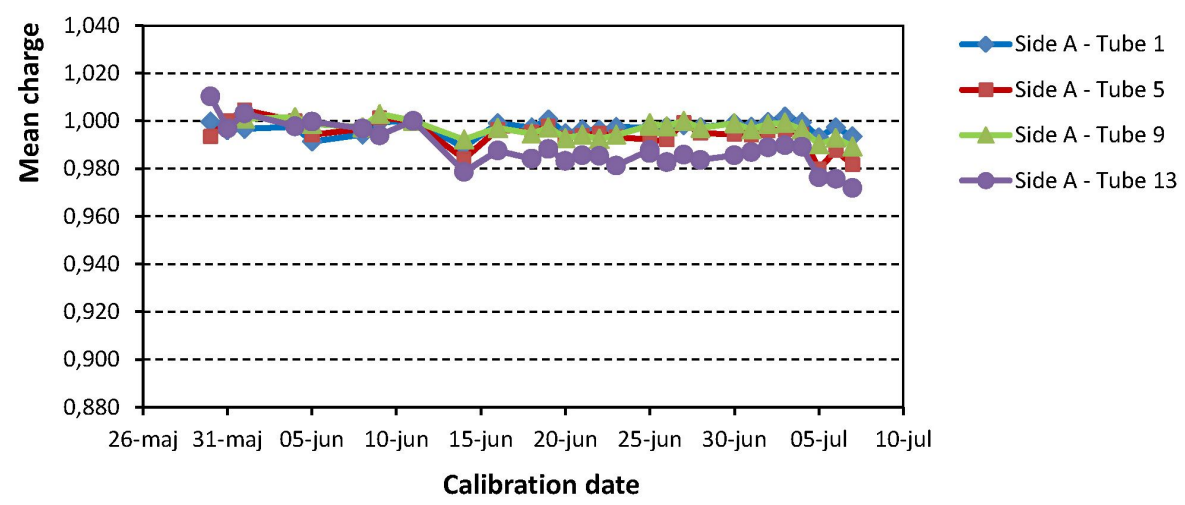

Figure 4: Measured mean charge in calibration runs using the Bi-207 sources. The charge is normalized to 1 for a run on the 11th of June. The mean charge is proportional to the gain of the photomultipliers. Taken from Ref. [3].

\section{Luminosity measurements}

Luminosity is measured by LUCID from a measurement of the number of PMT-hits, the number of bunch crossings with at least one PMT-hit and the integrated pulseheight (charge). These measurements are done over a time period called a luminosity block, which are typically 1 minute long and they are done for each of the individual bunch crossings in the LHC. The new electronics provides luminosity measurements using 124 different algorithms which take as input different combination of hits or charge from different tubes. Algorithms, which are based on PMT-hits from only one of the detectors (either A or C), are calculated by the LUCROD VME custom boards, 
while algorithms which depend on combination of hits from both detectors are calculated by the LUMAT boards (see Fig. 5). The luminosity is proportional to the measured charge which is in turn proportional to the logarithm of the measured number of PMT-hits. The two types of measurements therefore have different limitations. The main issue with the charge measurement is PMT gain stability while the hit measurements can suffer from pile-up of several signals below threshold giving a signal above threshold. Figure 6 shows the number of PMT-hits from different LHC bunches. The large peaks correspond to six trains of six colliding bunches each, plus two isolated colliding bunches. Two smaller peaks that correspond to bunches with only one beam are also seen. The baseline background level is due to the $\mathrm{Bi}-207$ source used for monitoring of the photomultiplier gain.

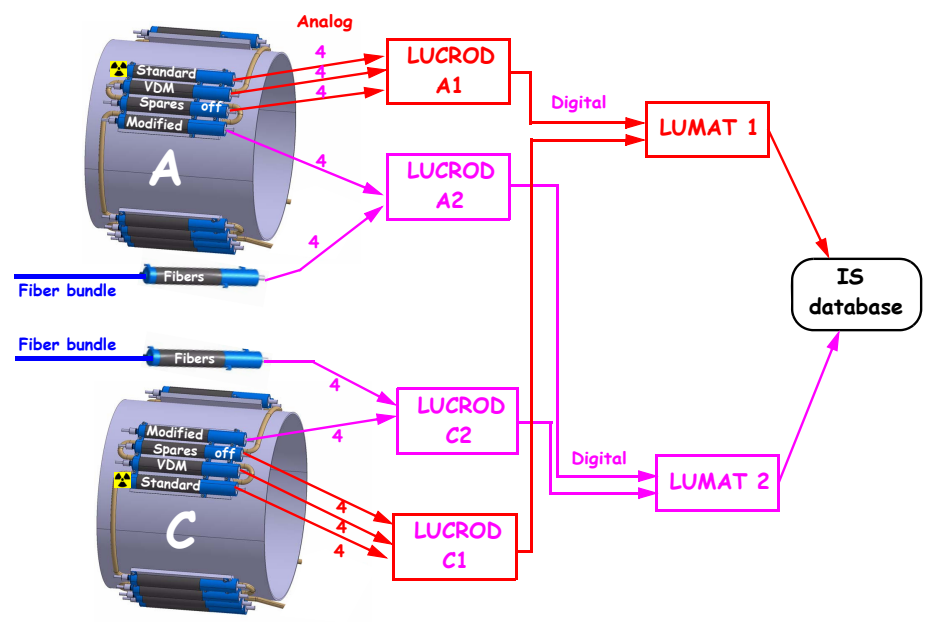

Figure 5: Bloc diagram of the LUCID electronics. Signals from all tubes are collected by 4 LUCROD cards (two per each side) that digitize the signals with FADCs. Some of the luminosity algorithms are calculated in the LUCRODs. Output of these calculations as well as a copy of all digitized PMT signals are sent to the LUMAT cards afterwards, which perform calculations of algorithms that combine data from both detectors and publish the results to the Information Server (IS) database.

\section{First $13 \mathrm{TeV}$ collisions at the LHC}

The LHC reported the first $13 \mathrm{TeV}$ pp collision in May of 2015 and these were recorded by ATLAS and other LHC experiments. Starting from that time the new LUCID was succesfully operating and provided information about the luminosity delivered to ATLAS. More than a femtobarn of luminosity is already recorded by ATLAS, which provides a sufficient amount of data about the performace of the LUCID detector with a high number of pp-interaction per bunch crossing.

The PMT pulseheight distribution in a physics run is shown in the left plot of Fig. 7 (blue) together with the same distribution during a Bi-207 calibration run (red). In both distributions a peak due to Cherenkov photons is visible. The calibration distribution is cut due to the threshold in the electronics that define a PMT-hit. 


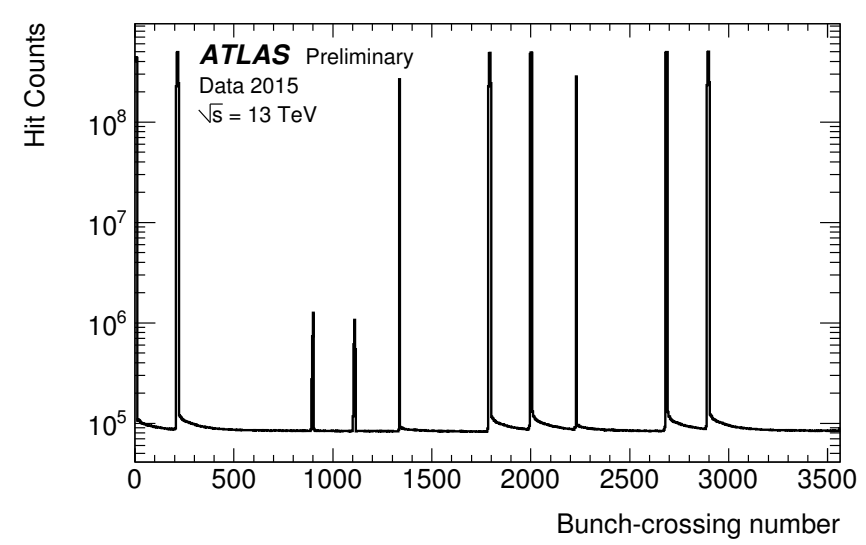

Figure 6: Bunch-by-bunch hit counts measured by a single LUCID photomultiplier as a function of the bunch-crossing number during a $13 \mathrm{TeV}$ run recorded on June 14, 2015 (LHC fill 3858). Taken from Ref. [3].

In the right plot of Fig. 7 a comparison of the pulseheight distributions in a physics run with low- $\mu$ (red) and high- $\mu$ (blue) are shown. The pulse height is shifted towards higher values when at high luminosity several particles traverse the photomultiplier window in the same bunch crossing.
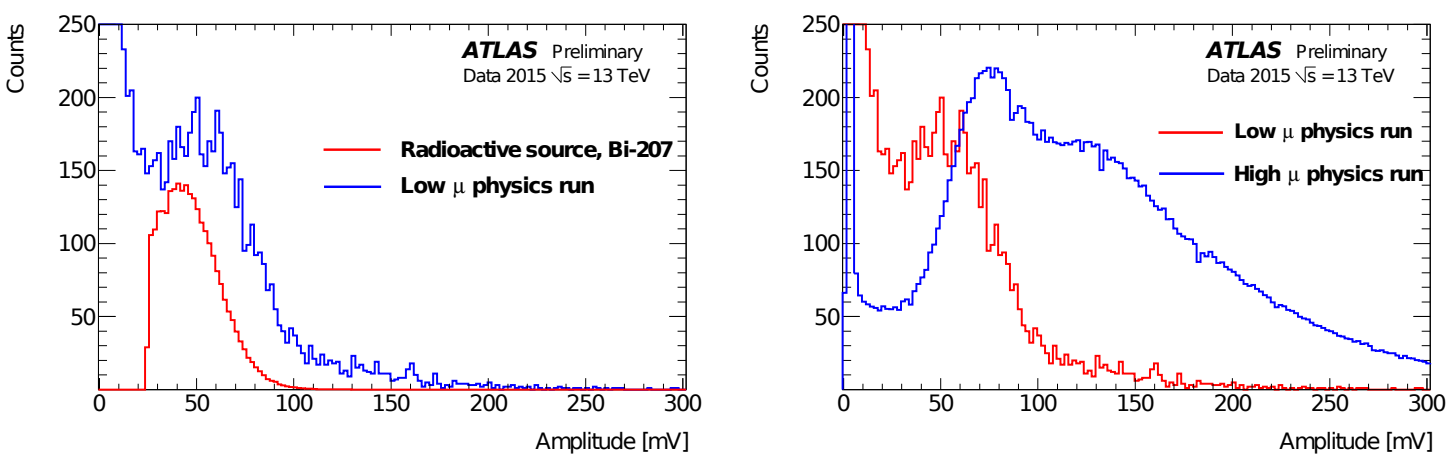

Figure 7: Comparison of pulseheight distribution in a physics run with low- $\mu$ with the same distribution during a Bi-207 calibration run (left) and with distribution with high- $\mu$ physics run. Taken from Ref. [3].

LUCID can measure luminosity in many ways and Fig. 8 shows a comparison of the luminosity measured by an $\mathrm{A}$ and a $\mathrm{C}$ detector for different ATLAS data taking runs. The two measurements agree to better than $0.5 \%$.

The left plot of Fig. 9 shows a measurement of the average number of inelastic pp collisions using different ATLAS luminometers and the right plot shows the ratio of this measurement with respect to a LUCID measurement. One of the detectors shows a deviation of up to $2 \%$ during this LHC fill but the other measurements are all in agreement with LUCID to better than $0.5 \%$.

The first month of data taking with the new detector therefore showed that LUCID could measure the relative luminosity with a precision of about $0.5 \%$. 


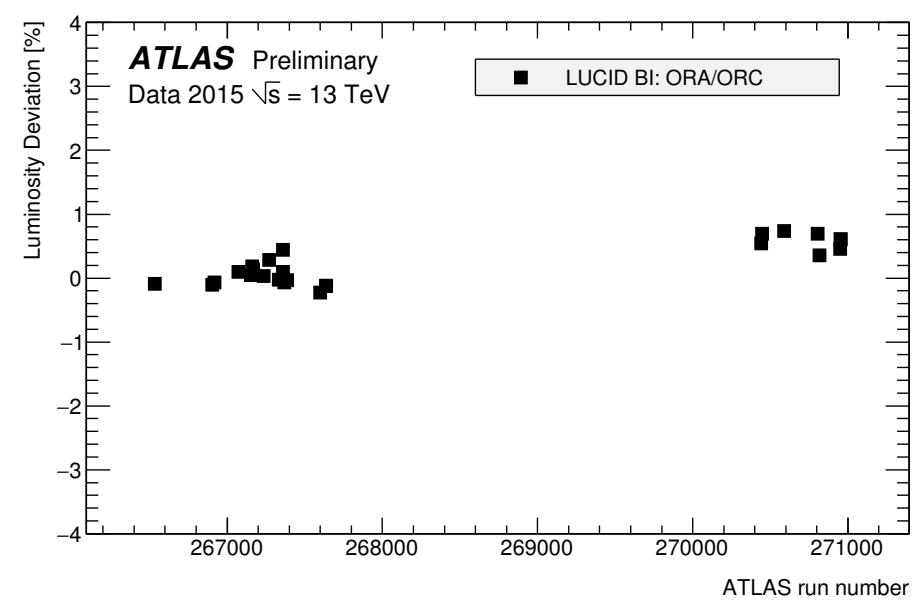

Figure 8: Fractional difference in measured luminosity between the forward (A) and backward (C) arms of the LUCID detector. The agreement between the two LUCID arms is better than $1 \%$. Taken from Ref. [3].
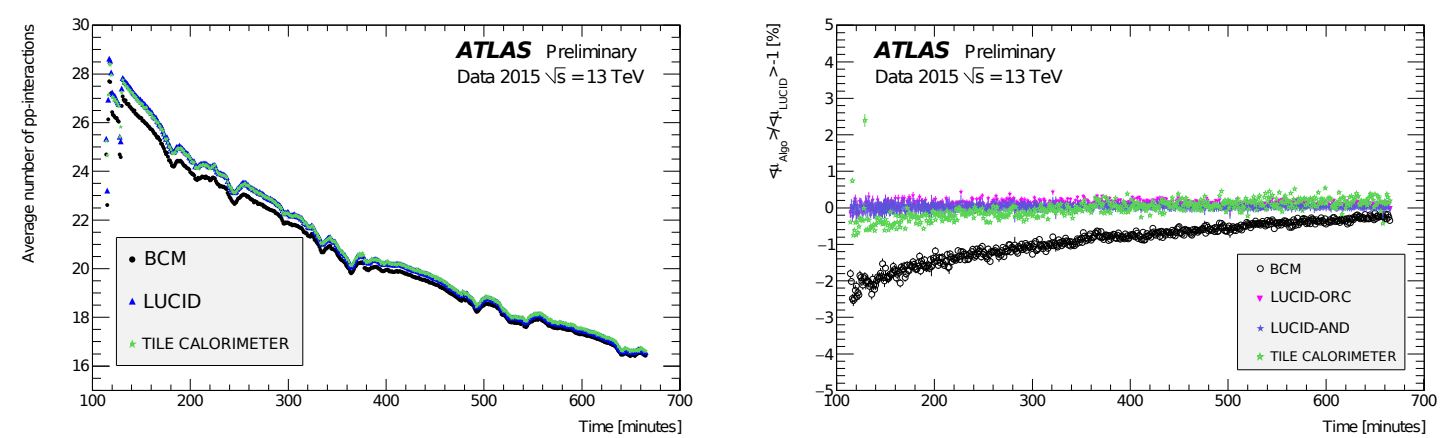

Figure 9: (left) Average number of inelastic proton-proton collisions per bunch crossing during a $13 \mathrm{TeV}$ fill; (right) Comparison of measured luminosity by different luminometers in ATLAS with respect to LUCID. Taken from Ref. [3].

\section{Conclusions}

A new LUCID detector has been installed in the ATLAS experiment in 2015. It has provided a high-precision measurement of the luminosity from the first day of data taking. The detector stability is monitored by three independent systems and the electronics provides up to 124 different luminosity measurements from different algorithms which use different type and combination of PMT signals. 


\section{References}

[1] ATLAS Collaboration, Improved luminosity determination in pp collisions at $\sqrt{s}=7$ TeV using the ATLAS detector at the LHC, Eur. Phys. J. C 73 (2013) 8, 2518 [arXiv:1302.4393 [hep-ex]].

[2] ATLAS Collaboration, The ATLAS Experiment at the CERN Large Hadron Collider, JINST 3, S08003, 2008.

[3] https://twiki.cern.ch/twiki/bin/view/AtlasPublic/

ForwardDetPublicResults\#LUCID_figures 